\title{
Evidence for feature perturbations from character misidentifications
}

\author{
GARVIN CHASTAIN \\ Boise State University, Boise, Idaho
}

\begin{abstract}
Evidence that features from characters in a briefly exposed display undergo perturbation has come from tasks requiring direct reports of feature locations. In the current experiments, a different approach was taken in which perturbations were inferred from incorrect name responses to letter and number stimuli. The relative positions of two segments (features), one of which was anchored so that its perturbation would be difficult, determined the name of the character that was to be reported. The procedure produced data which suggest that feature perturbations occur when characters are exposed briefly, and that these perturbations tend to move toward fixation.
\end{abstract}

Wolford (1975) argued that identification errors on briefly exposed arrays of characters are due to feature perturbations that tend to proceed toward fixation. Features from the more peripheral characters are mislocalized to mix with those of characters lying closer to fixation, and the resulting feature mix makes identification difficult. Wolford and Shum (1980) reported some direct evidence in support of this account. After each exposure, subjects attempted to report the position of a tick that bisected one side of each of five squares that extended into the visual periphery. For squares in the left visual field, a tick on the left side of a square tended to be mislocalized to the right side, and for squares in the right visual field, a tick on the right side tended to be mislocalized to the left side. Thus, the ticks, each of which was considered to be a feature, seemed to have a tendency to undergo perturbation toward fixation.

However, the results that Wolford and Shum observed could have been due to factors other than feature perturbations. If subjects saw a tick clearly but were not sure of its location, they may have assumed that it was closer to fixation than it actually was because they saw it so well. Subjects even may have tended to guess that a tick that was not seen was on the more foveal side of a square. Finally, the attempt to remember the positions of five ticks per presentation may not always have been successful, and when a position was forgotten, the preferred default response may have been the side of the square nearest fixation. To eliminate these possibilities, Chastain (1982) briefly exposed a single vertical tick that was superimposed on a series of vertical colored strips extending into the periphery in each visual field. Although subjects expected a tick to appear on each exposure, on a few randomly selected trials no tick was presented to determine which location subjects would tend to choose when a tick

I would like the thank Charles W. Eriksen and anonymous reviewers for their valuable comments on the original manuscript. Requests for reprints should be addressed to Garvin Chastain, Department of Psychology, Boise State University, Boise, ID 83725. was not seen (but was supposed to have been present). When ticks that were actually presented were mislocalized, the choice tended to be the next location nearer the central fixation dot than the correct one, whereas ticks that were not present tended to be assigned the most peripheral possible location. The predominance of feature perturbations toward fixation thus seems not to be caused by subjects' preference to guess a location near fixation when the feature is not seen or its correct location is forgotten. Subjects seem also not to guess a location near fixation when a tick is seen clearly (on the assumption that its image must be falling on an area in or close to the fovea). Mislocalizations tended to be only one location nearer fixation than the correct one, even when the tick appeared at one of the more peripheral locations.

New evidence concerning Wolford's (1975) model has been gathered and is the subject of the current report. Errors produced and analyzed were of the type that the model was designed to explain: misidentifications of briefly exposed characters. In Experiment 1, a long line with a thick segment at each end was presented symmetrically across fixation in a vertical or horizontal orientation on each exposure. Beside one of the thick segments of vertically oriented lines, an open curve appeared which, when combined with the segment, produced the lowercase letter $b$, $\mathrm{d}, \mathrm{p}$, or q. Beside one of the thick segments of horizontally oriented lines, a short vertical shape appeared which, when combined with the segment, produced L, J, F, or 7 . Examples are shown in Figure 1. Thick segments were placed at the ends of a long line to prevent them from being mislocalized. However, the open curve or vertical shape was not anchored in this way and thus could be mislocalized more easily. If the open curve were beside the upper portion of the thick segment above fixation and became mislocalized toward fixation, $p$ or $q$ would be misidentified as $b$ or $d$, respectively. If it were beside the lower portion of the thick segment above fixation and became mislocalized away from fixation, $b$ or $d$ would become $\mathbf{p}$ or $q$, respectively. The reverse pattern of misidentifications would occur with mislocalization when the 

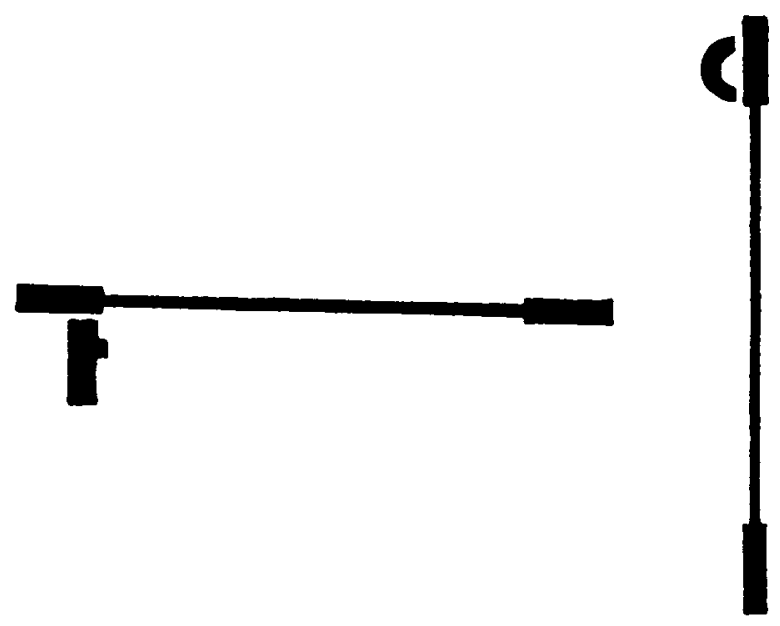

Figure 1. A sample horizontally oriented display producing the character 7 and a sample vertically oriented display producing the character $d$.

open curve was beside the thick segment below fixation. If the vertical shape were beside the left end of the thick segment to the left of fixation and became mislocalized toward fixation, $\mathrm{L}$ or $\mathrm{F}$ would become $\mathrm{J}$ or 7 , respectively. If the vertical shape were beside the right end of this same segment, mislocalization away from fixation would result in the misidentification of $\mathrm{J}$ or 7 as $\mathrm{L}$ or $\mathrm{F}$, respectively. The reverse pattern of misidentifications would occur with mislocalization if the vertical shape were beside the thick segment to the right of fixation. Stimuli with segments (features) that conjoin to produce a letter or character with a name were used to avoid requiring a direct localization response.

The pattern of errors in Experiment 1 was expected to suggest a predominance of feature perturbations moving toward fixation, in consonance with the predictions of Wolford's model. It was also expected that it might be possible to determine how much guessing bias contributed to the effects by analyzing errors placing the critical feature on the wrong side of the thick segment. An underlying assumption was that when this type of error occurs the critical feature is perceived very poorly or not at all and a guess is made regarding the character's identity.

\section{EXPERIMENT 1}

\section{Method}

Subjects. Twelve subjects served for extra credit in a general psychology course. Each reported normal or corrected-to-normal vision.

Apparatus. A three-channel Scientific Prototype tachistoscope, Model N-1000, was equipped with a microswitch to allow subjects to initiate exposures. The stimulus and fixation channels were illuminated to 130.0 and $65.0 \mathrm{~cd} / \mathrm{m}^{2}$, respectively, as measured by a Spectra Lumicon photometer with Photospot attachment.

Stimuli. Stimuli were traced onto white index cards in black ink with a Pilot Razor Point pen. A long vertical or horizontal line was traced using the slash on a Timesaver lettering guide, $760-\mathrm{V}$. It subtended a visual angle of approximately $.11^{\circ} \times 3.37^{\circ}$. Other portions of the stimuli were traced from an E-Z Letter plastic stencil, No. 81. A thick segment was the letter I, a vertical shape was the shaft of the number 4 , and the open curve was from the letter $P$, all in $1 / 2$-in. Gothic. The small spur on the vertical shape always pointed to the right and was on the upper half of the shape. Approximate visual angles subtended were: thick segment, $.21^{\circ} \times$ $.75^{\circ}$; open curve, $.27^{\circ} \times .54^{\circ}$, with an average stroke width of $.16^{\circ}$; and vertical shape, $.21^{\circ} \times .70^{\circ}$ (with the spur extending an additional $.11^{\circ} \times .16^{\circ}$ and beginning $.16^{\circ}$ from the top of the thick segment). An open curve or vertical shape was separated from the thick segment it was beside by a visual angle of approximately $.05^{\circ}$. The open curve and thick segment combined to produce the lowercase letter b, d, p, or q. The vertical shape and thick segment combined to produce the character $\mathrm{L}, \mathrm{J}, \mathrm{F}$, or 7 . A white fixation card was prepared with a black fixation dot, $.05^{\circ}$ in diameter, in the center. Cards were each taped to a Masonite slide for manual insertion into the tachistoscope.

Experimental Design. Half the subjects received the open curve and thick segment combinations; the remainder received the vertical shape and thick segment combinations. Each subject received 12 blocks of 16 trials per block. Within a block, the open curve or vertical shape was in each possible position on two presentations. The first two blocks were used for practice and duration setting, and were not analyzed. Order of presentations was completely randomized within each block.

Procedure. The instructions were to fixate the dot and press the microswitch at any time after a 200 -msec tone from the tachistoscope sounded to indicate that the stimulus slide was in place for that trial. The appearance of the stimulus slide interrupted the otherwise continuous presence of the fixation field. Immediately after the exposure, the subject reported the name of the letter or character produced by combining the open curve or vertical shape with the thick segment. The exposure duration was lowered during the first two blocks of trials from an initial setting of $100 \mathrm{msec}$ to allow approximately $70 \%$ accuracy. Thereafter the duration was changed only between blocks of trials to maintain that accuracy level. Trials proceeded in an uninterrupted series with no feedback regarding accuracy given any subject.

\section{Results and Discussion}

Two main types of errors were possible. Correct-side errors were cases in which the subject's response indicated a mislocalization of the critical feature which left it on the correct side of the thick segment. Incorrect-side errors were responses that indicated that the feature had been transposed to the wrong side of the thick segment. Correct-side errors were analyzed first. Shape beside the thick segment was analyzed as a between-subjects variable, direction from fixation of the character was nested within groups, and direction of mislocalization (inward toward fixation or outward away from fixation) of the critical feature as indicated by the incorrect response was analyzed as a within-subjects variable. The only significant effect was direction of mislocalization $[F(1,10)=5.50$, $p<.05$ ], with inward mislocalizations predominating. Means appear in Table 1.

The second analysis was of incorrect-side errors. Variables were the same as in the preceding analysis, except that direction of mislocalization had three levels: inward, outward, and at a correct distance from fixation. The only significant effect was direction of mislocalization $[F(2,20)=18.03, p<.001]$. Means appear in Table 1 . Orthogonal comparisons revealed no significant difference between the proportion of responses in error indicating 
Table 1

Mean Proportion of Responses That Were Correct- and Incorrect-Side Errors as a Function of the Direction of Mislocalization in Experiment 1

\begin{tabular}{ccc}
\hline $\begin{array}{c}\text { Direction of } \\
\text { Mislocation }\end{array}$ & \multicolumn{2}{c}{ Side of Thick Segment } \\
\cline { 2 - 3 } Correct & Incorrect \\
\hline Inward & .148 & .017 \\
Outward & .091 & .014 \\
Total & & .031 \\
None & & .047 \\
\hline
\end{tabular}

mislocalization inward and outward $(F<1)$ but a significant difference between the mean proportion of responses involved in these two types of error and the others $[F(1,20)=17.86, p<.01]$. However, since the analysis is of the proportion of all responses involved in each type of error, a more appropriate comparison would be between the sum of the proportion of errors indicating mislocalization inward and outward versus the other errors. This is because on each exposure yielding an incorrect-side error, a mistake indicating correct judgment of the critical feature's distance from fixation was possible (e.g., when $d$ was presented, b was always a possible error, and vice versa). On only half the exposures was each of the other two types of error possible (e.g., when $\mathrm{d}$ was presented, $\mathrm{p}$ but not $\mathrm{q}$ was a possible incorrect-side error; when $b$ was presented, $q$ but not $p$ was a possible incorrect-side error). The simple comparison proposed showed a significant difference $[t(11)=2.40, p<.05]$.

Exposure durations for subjects receiving the open curve and for those receiving the vertical shape were entered into a one-way analysis of variance. Those receiving the open curve required significantly longer exposures $[F(1,10)=22.56, p<.01]$. The means were $17 \mathrm{msec}$ for the open curve; and $7 \mathrm{msec}$ for the vertical shape. Studies have found that the retinal acuity gradient falls off more rapidly above and below the fovea than it does to the left and right (Schaller \& Dziadosz, 1975; Williams, 1984), and this may be the reason for the exposureduration difference. It may also be that the relative position of the open curve and thick segment was more difficult to judge than that of the vertical shape and thick segment.

When the critical feature that determined which of four lowercase letters or characters was present was judged to be on the correct side of the thick segment, errors indicating perturbation toward fixation were significantly more frequent than those indicating perturbation away from fixation. The absence of a significant interaction between direction of mislocalization and the betweensubjects variable of letter or character set or the nested variable of direction from fixation suggests that the effect was consistent across different conditions.

Responses indicating that the open curve or vertical shape was judged to be on the incorrect side of the thick segment apparently were not always caused by a complete absence of perception of these features. Significantly more often than not, subjects gave an incorrect response which placed the open curve or vertical shape at the cor- rect distance from fixation. For this reason, and because the proportion of responses involved in incorrect-side errors was very small, these responses provide little information about guessing biases.

In Experiment 1, the open curve or vertical shape did not physically touch the thick segment, but instead was separated from it slightly. The separation was $.05^{\circ}$, and under optimal conditions the minimum separable threshold for "normal" vision is around $.008^{\circ}$ (Alpern, Lawrence, \& Wolsk, 1967). When the separation was detected, it may have artificially encouraged or produced the perturbation of the separated feature. In Experiment 2, therefore, each open curve was physically touching the vertically oriented thick segment, and the long horizontal line had a circle on each end with a vertical bar attached to the side of one of the circles to produce b, $d, p$, or $q$ (some examples appear in Figure 2). As in Experiment 1, the pattern of misidentifications was expected to suggest the predominance of feature perturbations moving toward fixation.

\section{EXPERIMENT 2}

\section{Method}

Because of the similarity of the method to that of Experiment 1 , only the differences will be emphasized. Two groups of 8 new subjects per group served. Their essential characteristics were identical to those of subjects in the first experiment. The apparatus and
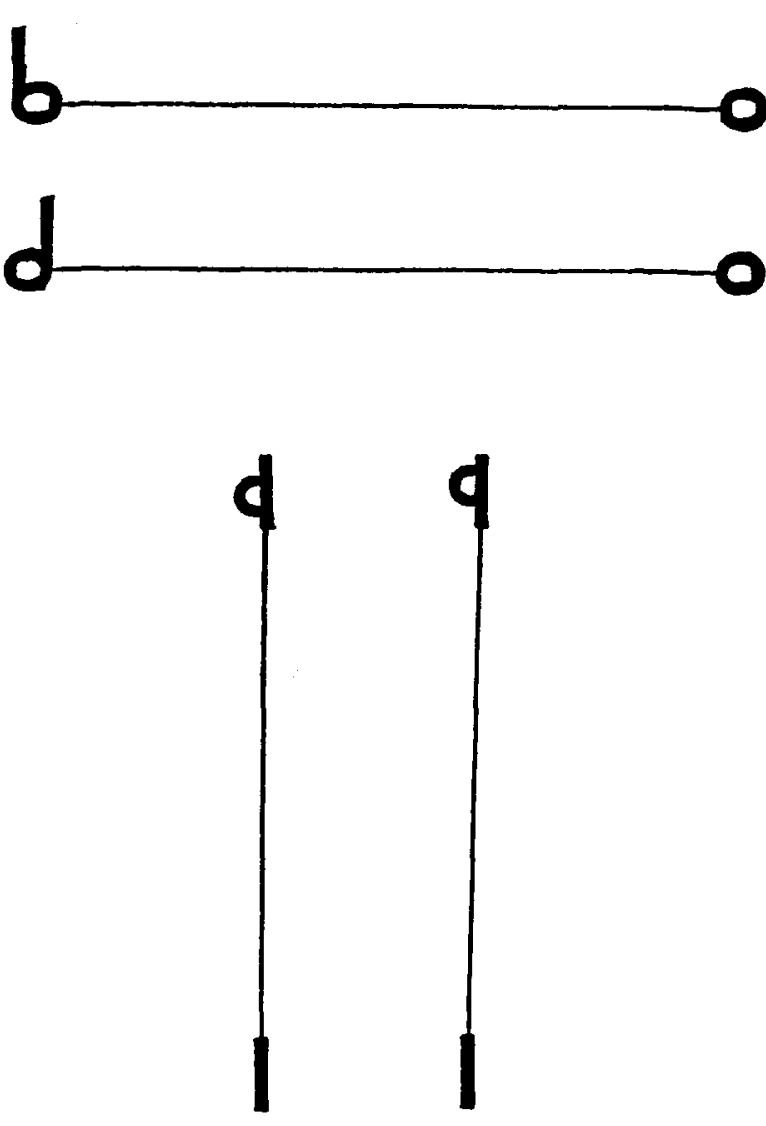

Figure 2. Four samples of displays that appeared in Experiment 2. 
its settings were those used in Experiment 1. Stimuli were traced onto cards with the pen and the Timesaver lettering guide used in that experiment, and the same fixation slide was used. The thin vertical lines were $3.64^{\circ}$ long and were centered horizontally. The thick segment at each end subtended a visual angle of approximately $.08^{\circ}$ $\times .54^{\circ}$; the open curve was $.19^{\circ} \times .29^{\circ}$, with its peripheral ex treme approximately $.08^{\circ}$ from the end of a thick segment. The peripheral extreme of the open curve was not made perfectly even with the end of the thick segment so that subjects could not use the alignment as a localization aid. The thin horizontal lines were $4.70^{\circ}$ long and were centered vertically. Flattened circles at each end of the thin horizontal lines were approximately $.32^{\circ} \times .27^{\circ}$ in visual angle. The vertical bars attached to circles were the same dimensions as the thick segments on the ends of the thin vertical lines. The vertical bars extended from the flattened circles in such a way that no corner beside a circle was present for possible use as an aid in localizing a bar. The experimental design was the same as that in Experiment 1. One group of subjects viewed characters that appeared above or below fixation; the other group viewed characters that appeared to the left or right. The procedure was that used for subjects who received the $b, d, p$, and $q$ combinations in Experiment 1. Exposure durations were adjusted during practice to allow each subject an overall accuracy of approximately $60 \%$; thereafter they were changed only between blocks to maintain this level of accuracy.

\section{Results and Discussion}

As in Experiment 1, both correct- and incorrect-side errors were possible for each subject, and correct-side errors were analyzed first. Critical feature to be localized in identifying the letter (open curve or vertical bar) was analyzed as a between-subjects variable, direction from fixation of the character was nested within groups, and direction of mislocalization of the critical feature as indicated by the incorrect response was analyzed as a withinsubjects variable. All main effects and interactions were significant beyond the .01 level except for the interaction between the nested variable of direction from fixation and direction of mislocalization $(p>.10)$. Means for the significant effects, except for the main effect of the nested variable, appear in Table 2. Planned comparisons indicated that inward mislocalizations predominated over outward ones when the critical feature was the open curve $[t(7)=20.20, p<.001]$ and when it was the vertical bar $[t(7)=4.21, p<.01]$. Although the difference in the overall proportion of responses in error to characters appearing above $(.316)$ versus below $(.370)$ fixation was not significant, a significantly higher proportion of responses were in error to characters appearing to the left of fixation (.219) than to those appearing to the right $(.142)[t(7)=4.86, p<.01]$.

Table 2

Mean Proportion of Responses That Were Correct-Side Errors as a Function of Critical Feature and Direction of Mislocalization in Experiment 2

\begin{tabular}{lccc} 
& \multicolumn{3}{c}{ Direction of Mislocalization } \\
\cline { 2 - 4 } Critical Feature & Inward & Outward & Mean \\
\hline Open Curve & .630 & .056 & .343 \\
Vertical Bar & .217 & .144 & .181 \\
Mean & .424 & .100 & \\
\hline
\end{tabular}

Table 3

Mean Proportion of Eligible Responses Involved in Incorrect-Side Errors in Experiment 2

Location of Characters Relative to Fixation

\begin{tabular}{lccccc}
\hline Above & Below & Mean & Left & Right & Mean \\
\hline 116 & .071 & .094 & .289 & .155 & .222 \\
\hline
\end{tabular}

The next analysis was of incorrect-side errors. Variables were those entered into the preceding analysis, although direction of mislocalization could be inward, outward, or none (at a correct distance from fixation). The last type of error was possible upon every exposure, yet each of the other two was possible on only half the exposures; proportions entered into the analysis were therefore proportion of eligible exposures involved in each type of error. Each of the three main effects was significant beyond the .01 level, but neither interaction was significant $(F \mathrm{~S}<1)$. Means for the effect of groups and the nested variable appear in Table 3. An orthogonal comparison showed that the proportion of eligible responses in error placing the critical feature at the correct distance from fixation (.187) was not quite significantly different from the mean of the proportion placing the feature nearer fixation than it lay (.167) and farther from fixation than it lay $(.119)[F(1,14)=3.87, .05<p<.10]$. The other permissible orthogonal comparison, testing the latter two means, yielded a significant difference $[F(1,14)=7.51$, $p<.02]$.

Exposure durations for subjects identifying characters above and below fixation were compared with those for the other subjects. The former group required significantly longer exposures $[t(14)=3.35, p<.01]$. The means were $11 \mathrm{msec}$ for those receiving characters above or below fixation and $6 \mathrm{msec}$ for those receiving characters to the left or right of fixation.

As in Experiment 1, for critical features judged to be on the correct side of the standard, as indicated by the subject's response, errors suggesting perturbation toward fixation were significantly more frequent than those suggesting perturbation away from fixation. The critical feature to be localized was not the same for the groups. The initial analysis showed that although there was no significant difference in performance on characters appearing above and below fixation, those appearing to the left were identified significantly less accurately than those appearing to the right. This is consistent with the results of other studies that have shown a right visual field advantage for the identification of unilaterally displayed alphabetic material (e.g., Bryden, 1966). Although this advantage was not significant in Experiment 1, the smaller number of subjects in that experiment may have made it more difficult to observe.

Even with incorrect-side errors, responses tended to place the critical feature more often at the correct distance from fixation than at the incorrect distance. It seems, therefore, that subjects were not always merely guessing 
when they made an incorrect-side error, but often had some information about the distance of the critical feature from fixation. The critical feature was mislocalized inward significantly more frequently than it was mislocalized outward. The pattern of misjudgments of the distance of the critical feature from fixation was similar for correctand incorrect-side errors. However, since the distance usually was not misjudged, incorrect-side errors provide little information about guessing biases.

In the current experiment, as in Experiment 1, the distance from fixation of the critical feature was not held constant. Each critical feature could be at two possible distances from fixation. When a critical feature was in the peripheral location, there might have been more errors because it was more difficult to see. More incorrect reports with the critical feature in the peripheral location is subject to misinterpretation as feature perturbations moving predominantly toward fixation. Such an explanation seems implausible for several reasons. First, Chastain (1982) found that features that were not perceived tended to be assigned the most peripheral possible location. Poor perception of the critical feature in the peripheral location would therefore not seem to be the basis of the asymmetry that was observed. Also, inward mislocalizations were much more prevalent with the open curves than with the vertical bars. The difference in distance between their two locations was about 2.7 times greater for the vertical bars $\left(.24^{\circ}\right)$ than for the open curves $\left(.09^{\circ}\right)$. If the apparent predominance of inward mislocalizations was due to more errors when a critical feature was at the peripheral location, the effect should increase with increases in the difference in distance between the possible locations. Instead, inward mislocalizations were more prevalent when the difference was smaller. In addition, the vertical bars were, on the average, 1.2 times farther from fixation than the open curves $\left(2.51^{\circ}\right.$ vs. $\left.2.09^{\circ}\right)$. An explanation relating the effect to greater difficulty in seeing a critical feature in the peripheral location again would predict more inward mislocalizations with the vertical bars. Finally, incorrect-side errors were more frequent with the vertical bars, suggesting that perhaps they were generally more difficult to see, yet inward mislocalizations were more frequent with the closed curves.

In addition to their distance from fixation and the distance between their possible locations, other factors regarding the critical features were not held constant. The open curves may have been more difficult to localize because of their size (they did not extend as far from the part of the letter attached to the thin line as the vertical bars did). The open curves were probably more subject to perturbation than the vertical bars because the distance between their possible locations was smaller. The current results therefore do not yield information about the relative difficulty of localizing features with different shapes.

As in Experiment 1, subjects who viewed characters that appeared above and below fixation required a longer exposure duration to maintain the desired overall accuracy level than did those who viewed characters that appeared to the right and left of fixation. The explanation proposed in Experiment 1 regarding acuity differences for material in different parts of the visual field may be relevant to this effect.

\section{GENERAL DISCUSSION}

The two foregoing experiments were consistent in their support of predictions stemming from Wolford's (1975) feature perturbation model. Characters appearing in the visual periphery contained features that could be mislocalized relative to one another. Subjects were required not to localize the features directly, but instead to identify the character composed of those features (e.g., "b," "d,", "q," "p"). The pattern of incorrect responses suggested that features tended to be mislocalized toward fixation. This pattern was consistent between two experiments and across four directions from fixation and two character sets. In Experiment 1, features of the characters were physically separated by a short distance; in Experiment 2, the features were connected as they would normally be in a typed or printed character. Although differences in the size and positioning of the features in Experiment 2 yielded more variation in the types of errors between the character sets, the pattern of inward mislocalizations was as apparent with each character set as it had been in Experiment 1 when the features were not physically connected. Although guessing biases were difficult to assess, the predominance of inward mislocalizations does not seem to be due merely to more localization errors with increases in the distance of the critical feature from fixation.

Besides Wolford's theory, another type of theory proposes that errors in recognizing characters may result from failures to conjoin features correctly. In their featureintegration theory of attention, Treisman and Gelade (1980) argued that features outside the range of focal attention will often be integrated in ways that produce incorrect conjunctions. Their theory stresses perceptual grouping on the basis of attended versus unattended features rather than direction or distance in predicting the nature of feature mislocalizations. In a series of experiments, Treisman and Schmidt (1982) found that the color, shape, size, and solidity of the letters and forms they used could be conjoined incorrectly. However, whether the features were aligned toward fixation either was not controlled or its effect generally was not reported. Nevertheless, in one of their experiments, three letters appeared on each exposure with the middle one at fixation, and the researchers commented that a frequent error was for subjects to mislocalize one feature from each of the outer letters, reporting the resulting misconjunction as being at the location of the middle letter. Wolford's (1975) theory would predict just this type of frequent mislocalization toward fixation. Further research of the type in which stimulus dimensions and alignment toward fixation are 
systematically varied may provide evidence that allows different theories such as those of Treisman and Gelade (1980) and Wolford (1975) to be integrated.

\section{REFERENCES}

AlPeRn, M., LAWRENCE, M., \& WolsK, D. (1967). Sensory processes. Belmont, CA: Brooks/Cole.

BRYDEN, M. P. (1966). Left-right differences in tachistoscopic recognition: Directional scanning or cerebral dominance? Perceptual \& Motor Skills, 23, 1127-1134.

Chastain, G. (1982). Feature mislocalizations and misjudgments of intercharacter distance. Psychological Research, 44, 51-66.

Schaller, M. J., \& Dziadosz, G. M. (1975). Individual differences in adult foveal vision asymmetries. Journal of Experimental Psychology: Human Perception \& Performance, 1, 353-365.

Treisman, A., \& Gelade, G. (1980). A feature integration theory of attention. Cognitive Psychology, 12, 97-136.

Treisman, A., \& SCHMidT, J. (1982). Illusory conjunctions in the perception of objects. Cognitive Psychology, 14, 107-141.

WILLIAMS, L. J. (1984). Information processing in near peripheral vision. Journal of General Psychology, 111, 201-207.

Wolford, G. (1975). Perturbation model for letter identification. Psychological Review, 82, 184-199.

Wolford, G., \& ShUm, K. H. (1980). Evidence for feature perturbations. Perception \& Psychophysics, 27, 409-420.

(Manuscript received August 21, 1985; revision accepted for publication April 2, 1986.) 